\title{
Comparison of a polyethylene glycol membrane and a collagen membrane for the treatment of bone dehiscence defects at bone level implants-A prospective, randomized, controlled, multicenter clinical trial
}

Jung, Ronald Ernst ; Mihatovic, Ilja ; Cordaro, Luca ; Windisch, Péter ; Friedmann, Anton ; Blanco Carrion, Juan ; Sanz Sanchez, Ignacio ; Hallman, Mats ; Quirynen, Marc ; Hammerle, Christoph H F

\begin{abstract}
OBJECTIVES The aim of the present randomized, controlled clinical multicenter trial was to compare a polyethylene glycol (PEG) and a native collagen membrane (BG) for simultaneous guided bone regeneration at bony dehiscence-type defects around bone level titanium implants. MATERIAL AND METHODS The study enrolled 117 patients requiring implant treatment in the posterior maxilla or mandible with expected buccal bony dehiscence-type defects at the placed titanium implants. According to a parallel groups design, defects were filled with a synthetic bone filler and randomly assigned to either PEG or BG membrane. As primary parameter, the relative vertical bone fill was assessed at baseline and at re-entry after 6 months of healing. As secondary parameters, the marginal bone level (MBL) was assessed radiographically and soft tissue conditions were recorded up to 18 months postloading. RESULTS Both groups showed comparable vertical bone fill revealing a relative change in defect height of $59.7 \%$ (PEG) and $64.4 \%$ (BG). The absolute mean reduction in defect size was $2.5 \mathrm{~mm}$ in the PEG group and $3.2 \mathrm{~mm}$ in the BG group. Although both groups revealed a statistically significant mean defect reduction $(\mathrm{p}<.001)$, a comparison between the two groups did not show statistical significances. The non-inferiority test with inferiority limit of $-5 \%$ could not be rejected, based on the $90 \%$ confidence interval of the differences of the two means with lower limit -15.4\%. After 18 months, an MBL increase of 0.45 $\pm 0.43 \mathrm{~mm}$ in the PEG group and $0.41 \pm 0.81 \mathrm{~mm}$ in the BG group was detected $(\mathrm{p}<.001)$. Soft tissue complications were observed in both groups without showing statistical significance. CONCLUSIONS Both membranes supported bone regeneration at dehiscence-type defects and obtained vertical bone fill with a relative change in defect height of $59.7 \%$ (PEG) and $64.4 \%$ (BG); however, the non-inferiority of PEG could not be shown.
\end{abstract}

DOI: https://doi.org/10.1111/clr.13657

Posted at the Zurich Open Repository and Archive, University of Zurich

ZORA URL: https://doi.org/10.5167/uzh-194797

Journal Article

Accepted Version

Originally published at:

Jung, Ronald Ernst; Mihatovic, Ilja; Cordaro, Luca; Windisch, Péter; Friedmann, Anton; Blanco Carrion, Juan; Sanz Sanchez, Ignacio; Hallman, Mats; Quirynen, Marc; Hammerle, Christoph H F (2020). Comparison of a polyethylene glycol membrane and a collagen membrane for the treatment of bone dehiscence defects at bone level implants-A prospective, randomized, controlled, multicenter clinical trial. Clinical Oral Implants Research, 31(11):1105-1115. 
DOI: https://doi.org/10.1111/clr.13657 


\title{
Comparison of a polyethylene glycol membrane and a collagen membrane
}

for the treatment of bone dehiscence defects at bone level implants - a prospective, randomized, controlled, multicenter clinical trial

Jung RE ${ }^{\mathrm{a}^{*}}$, Mihatovic $\mathrm{I}^{\mathrm{b}}$, Cordaro $L^{\mathrm{c}}$, Windisch $\mathrm{P}^{\mathrm{d}}$, Friedmann $A^{\mathrm{e}}$, Blanco Carrion $\mathrm{J}^{\mathrm{f}}$ Sanz Sanchez Ig, Hallman $M^{\mathrm{h}}$, Quirynen $\mathrm{M}^{\mathrm{i}}$, Hammerle $\mathrm{CHF}$ a

${ }^{a}$ Clinic of Reconstructive Dentistry, University of Zurich, Plattenstrasse 11, 8032 Zurich, Switzerland

${ }^{b}$ Department of Oral Surgery, Heinrich Heine University, Düsseldorf, Germany

${ }^{\mathrm{C}}$ Department of Periodontics and Prosthodontics, Policlinico Umberto I, Eastman Dental Hospital, Rome, Italy

${ }^{d}$ Department of Periodontology, Semmelweis University, Budapest, Hungary

e Department of Periodontology, School of Dentistry, Faculty of Health, Witten/Herdecke University, AlfredHerrhausen-Str. 50, 58455 Witten, Germany

${ }^{f}$ Periodontology Unit, School of Medicine and Dentistry, University of Santiago de Compostela, Santiago de Compostela, Spain

${ }^{g}$ ETEP (Etiology and Therapy of Periodontal Diseases) Research Group, University Complutense, Madrid, Spain.

${ }^{h}$ Department of Oral and Maxillofacial Surgery, Public Health Service, Gävle, Sweden

i Section of Periodontology, Department of Oral Health Sciences, KU Leuven, Leuven, Belgium.

\section{Corresponding address:}

\author{
Prof. Dr. Ronald E. Jung; PhD \\ University of Zurich, Center of Dental Medicine \\ Clinic of Reconstructive Dentistry \\ Plattenstrasse 11 \\ $\mathrm{CH}-8032$ Zurich, Switzerland \\ Phone: +41446343252 \\ Fax: +41446344305 \\ e-mail: ronald.jung@zzm.uzh.ch
}

Short title: Comparison of a polyethylene glycol membrane and a collagen membrane for the treatment of bone dehiscence defects

Key words: Guided bone regeneration, barrier membranes, bone substitutes, alveolar ridge defect 


\section{Source of Funding}

The study was funded by Institute Straumann AG, Basel, Switzerland. The study materials were provided by Institut Straumann AG.

\section{Conflict of Interests}

The authors have received lecturing fees and funds for research project by Institute Straumann AG, Basel, Switzerland.

*Jung RE and Mihatovic I are considered as joint first authors

\section{Author contributions}

\section{Contribution role according to the CRediT Taxonomy}

\begin{tabular}{|l|l|l|}
\hline 1) Conceptualization & 6) Methodology & 11) Validation \\
\hline 2) Data Curation & 7) Project administration & 12) Visualization \\
\hline 3) Formal analysis & 8) Resources & 13) Writing-original draft \\
\hline 4) Funding acquisition & 9) Software & 14) Writing-review \& editing \\
\hline 5) Investigation & 10) Supervision & \\
\hline
\end{tabular}

$\begin{array}{ll}\text { Jung RE } & 1,2,3,4,5,6,7,8,9,10,11,12,13,14 \\ \text { Mihatovic } & 5,12,13,14 \\ \text { Cordaro L } & 5,14 \\ \text { Windisch P } & 5,14 \\ \text { Friedmann A } & 5,14 \\ \text { Blanco Carrion J } & 5,14 \\ \text { Sanz Sanchez I } & 5,14 \\ \text { Hallmann M } & 5,14 \\ \text { Quirynen M } & 5,14 \\ \text { Hämmerle CHF } & 1,2,3,4,5,6,7,8,10,11,14\end{array}$




\section{Abstract}

Objectives: The aim of the present randomized, controlled clinical multicenter trial was to compare a polyethylenglycol (PEG) and a native collagen membrane (BG) for simultaneous guided bone regeneration (GBR) at bony dehiscence-type defects around bone level titanium implants.

Material and methods: The study enrolled 117 patients requiring implant treatment in the posterior maxilla or mandible with expected buccal bony dehiscence-type defects at the placed titanium implants. According to a parallel groups design, defects were filled with a synthetic bone filler and randomly assigned to either PEG or BG membrane. As primary parameter, the relative vertical bone fill was assessed at baseline and at re-entry after 6 months of healing. As secondary parameters, the marginal bone level (MBL) was assessed radiographically and soft tissue conditions were recorded up to 18 months post-loading.

Results: Both groups showed comparable vertical bone fill revealing a relative change in defect height of $59,7 \%$ (PEG) and $64,4 \%$ (BG). The absolute mean reduction in defect size was $2.5 \mathrm{~mm}$ in the PEG group and $3.2 \mathrm{~mm}$ in the BG group. Although both groups revealed a statistically significant mean defect reduction $(p<0.001)$, a comparison between the two groups did not show statistical significances. The non-inferiority test wih inferiority limit of $-5 \%$ could not be rejected, based on the $90 \%$ confidence interval of the differences of the two means with lower limit $-15.4 \%$. After 18 months, an MBL increase of $0.45 \pm 0.43 \mathrm{~mm}$ in the PEG group and $0.41 \pm 0.81 \mathrm{~mm}$ in the BG group was detected $(p<0.001)$. Soft tissue complications were observed in both groups without showing statistical significance.

Conclusions: Both membranes supported bone regeneration at dehiscence-type defects and obtained vertical bone fill with a relative change in defect height of 59.7\% (PEG) and $64.4 \%$ (BG), however the non-inferiority of PEG could not be shown. 


\section{Introduction}

Guided bone regeneration (GBR) has become a standard procedure to treat alveolar ridge defects (Jensen \& Terheyden, 2009). At dehiscence-type defects around dental implants a simultaneous application of bone filler materials such as autografts, xenografts or allografts in combination with a barrier membrane improved clinical outcomes (Benic \& Hammerle, 2014; Jensen \& Terheyden, 2009). The application of a barrier membrane stabilizes particulated grafts in the defect and allows bone regeneration within the augmented area without being inhibited by the migration of faster proliferating connective tissue cells (Gottlow, Laurell, Teiwik \& Genon, 1994; Nyman \& Lang, 1994). Currently, the use of different synthetic and nonsynthetic biodegradable barrier membranes show promising clinical long-term outcomes (Jung, Benic, Scherrer \& Hammerle, 2015; Jung, Fenner, Hammerle \& Zitzmann, 2013; Ramel, Wismeijer, Hammerle \& Jung, 2012). Native collagen supports early tissue integration and transmembraneous angiogenesis, but was characterized by a fast biodegradation (Herten, et al., 2009; Rothamel, et al., 2005; Schwarz, Rothamel, Herten, Sager \& Becker, 2006). Recently, a resorbable polyethylenglycol (PEG) membrane was introduced being associated with promising preclinical (Herten, et al., 2009; Jung, et al., 2006; Mihatovic, Becker, Golubovic, Hegewald \& Schwarz, 2012; Schwarz, Mihatovic, Golubovic, Hegewald \& Becker, 2012a) and clinical results (Jung, Halg, Thoma \& Hammerle, 2009). The in-situ gelling polyethylenglycol membrane can be applied individually according to the defect configuration and stabilizes the bone graft material in the desired shape. Preclinically, the application of a polyethylenglycol membrane supported bone regeneration and osseointegration of titanium implants after either simultaneous or staged GBR and implant placement (Jung, et al., 2009; Mihatovic, et al., 2012; Schwarz, et al., 2012a). Clinically, a randomized clinical trial investigated simultaneous GBR at dehiscence-type defects around titanium implants using a polyethylenglycol membrane or a native collagen membrane together with a deproteinized bovine bone material. Both membrane types demonstrated comparable vertical bone fill in both groups of $95-96 \%$. Moreover, a more favorable clinical handling was noted for the test device (Jung, et al., 2009). Based on the promising data, the aim of the present study was to compare 
the polyethylenglycol membrane and a native collagen membrane for the treatment of bony dehiscence-type defects around titanium implants in a prospective, controlled, randomized, clinical multicenter trial.

\section{Material and Methods}

\section{Study design}

The present study was designed as a prospective, randomized, controlled multicenter clinical investigation evaluating the amount of vertical bone fill in dehiscence-type defects using a polyethylenglycol membrane or a native collagen membrane. Prior to the start of the study, an investigator calibration meeting was held to aim for consensus regarding the study protocol by all 9 European centers. The 9 study centers were both, University (6 centers) and private practice ( 3 centers) based. They were well distributed throughout Europe and were located in Zürich (Switzerland), Düsseldorf (Germany), Rome (Italy), Budapest (Hungary), Berlin (Germany), Santiago de Compostela (Spain), Madrid (Spain), Gävle (Sweden) and Leuven (Belgium). Following approval by the respective local ethics committees in all 9 centers, 117 patients were recruited for the study. Informed consent was obtained from all patients prior to the start of the investigation.

\section{Randomization}

An independent statistician generated a randomization list based on one GBR treatment per patient for a total of 118 surgical sites. Further randomization lists of patients were generated to account for patients found ineligible at surgery. Patients were enrolled sequentially. Randomization envelopes were supplied to each centre and numbered sequentially containing the treatment allocation according to the randomization list. Investigators should identify eligible patients during the baseline visit and a number was allocated by the order of entry into the study. During surgery at visit 2 the randomization envelope was opened after flap elevation and implant placement revealing a buccal bony dehiscence defect. The investigator filled out 
the randomization page of the CRF and faxed it to the monitor. In case that the respective patient was found ineligible, a new patient was recruited and a new randomization number was selected with a new envelope.

\section{Patients}

A total of $n=117$ patients were recruited in 9 European dental clinics. The mean age of the patients was 48.7 years ( \pm 14.3 years) ranging from 19 to 77 years $(68.2 \%$ female vs. $31.8 \%$ male) (table 1). A clinical examination was performed when a patient met all local or systemic study inclusion and exclusion criteria. Inclusion criteria, such as primary stability of the implant and vertical dehiscence defect of $>3 \mathrm{~mm}$ was evaluated at the day of surgery.

All the following criteria had to be met for inclusion in the study:

1) Subjects must have voluntarily signed the informed consent form before any study related action

2) Patients must be at least 18 years of age and not more than 80 years old.

3) Have at least one missing tooth in the posterior mandible or maxilla in quadrant 1, 2, 3, 4 (FDI positions 4-7), requiring one or more dental implants.

4) Partially edentulous patients and patients in need of a fixed dental prosthesis

5) The tooth at the implant site(s) must have been extracted or lost at least 6 weeks before the date of implantation.

6) Bone defect(s) must be present with a vertical dimension greater than or equal to $3 \mathrm{~mm}$ at the foreseen GBR site measured after dental implant placement.

7) Full mouth bleeding on probing (FMBoP) and full mouth plaque score (FMPI) are both lower or equal than $25 \%$

8) Patients must be committed to the study

The following systemic exclusion criteria disqualified patients from the study:

1) Systemic disease

2) Use of steroids

3) Pregnancy 
4) Physical handicaps interfering with performance of oral hygiene

5) Intravenous bisohosphates

6) Alcoholism or chronic drug abuse

7) Immune compromise

8) Smoking of more than 10 cigarettes per day

The following local exclusion criteria disqualified patients from the study:

1) local infection or untreated periodontitis

2) Regenerative treatment adjacent to the planned study site of interest

3) Mucosal desease or oral lesion

4) History of local irradiation therapy

5) Severe bruxing / clenching habits

6) Previous regenerative treatment at the planned study site of interest

\section{Primary parameter}

The relative vertical bone fill was assessed by probing at baseline and compared to re-entry after 6 months of healing. It was measured mid-buccaly as the distance from the first bone-toimplant contact to the implant shoulder using a calibrated periodontal probe (Fig. 1).

\section{Secondary parameters}

The following secondary parameters were assessed:

Crestal bone level changes by means of periapical radiographs (at baseline, implant loading and follow-up visits after 18 months). The radiographs were evaluated by a single reader using a calibrated measuring tool. The reference point for bone level measurements was the implant shoulder. Bone level changes were evaluated by measuring the distance between the mesial and distal implant shoulder and the first visible bone-to-implant contact. Additionally, the following soft tissue parameters were evaluated: physical appearance and tissue biotype, the occurrence of soft tissue dehiscences, gingival recession as well as changes in the height of 
the keratinized gingiva. Soft tissue conditions were assessed during study visits at day 1 , day 7-10, 4 weeks, 3 months and 6 months. In addition, implant survival was calculated over the period of 18 months.

\section{Surgical procedure}

Prophylactic antibiotic therapy could be given at the discretion of the investigator and was recorded. Each patient received the study device using routine surgery. Surgery was performed under local anaesthesia following the respective centres standard surgical techniques. This visit comprised of the following phases: pre-surgery assessments, implant placement, GBR procedure and postoperative procedures.

\section{Implant placement}

If more than one site was eligible after the measurements, the most mesial site was chosen as site of interest. If more than one site was most mesial, the tooth position in the highest quadrant (FDI) was chosen. Subsequently, bone level titanium implants (Straumann BL, Institut Straumann AG, Basel, Switzerland) were inserted utilizing diameters (3.3 and $4.1 \mathrm{~mm}$ ) and lengths $(8,10$ and $12 \mathrm{~mm})$ according to the individual clinical and radiological situation. Immediately after implant placement, initial implant stability was assessed by hand testing. If implant at the study site of interest lacked primary stability at this assessment, the patient was not randomized and excluded from further participation in the study receiving alternative treatment. The bone defect of eligible sites was determined after implant placement measured from the first bone-to-implant contact to the implant shoulder with the help of a periodontal probe (Fig. 1b). If the defect height was $<3 \mathrm{~mm}$, the patient was excluded from the study. If the defect height was $\geq 3 \mathrm{~mm}$, the site was assigned to a treatment according to the randomization envelope. Additionally, the defect width $(\mathrm{mm})$ was measured horizontal at the height of the implant shoulder and it was assessed if the implant was inside or outside the bony envelope.

\section{GBR procedure}


After implantation, the osseous defects around the implants were augmented with a bone filler (alloplastic biphasic calcium phosphate, Straumann BoneCeramic (SBC), Institut Straumann AG, Basel, Switzerland) (Fig.2) and covered with either a test or a control membrane (Fig.2 a and $b$ )

- Control sites (native porcine-derived type I and type III collagen membrane (BG) (Geistlich BioGide, Geistlich Biomaterials AG, Wolhusen, Switzerland): The size of the membrane were adapted by scissors according to the exposed bone defect. The membrane overlapped the walls of the defect by at least $2 \mathrm{~mm}$ to allow complete bone contact and to prevent gingival connective tissue invasion below the material (Fig.2a)

- Test sites (polyethylenglycol membrane (PEG) (Straumann MembraGel, Straumann AG, Basel, Switzerland): The defect was covered with the PEG membrane. The mixed gel had to be applied directly over the bone defect using the dental tip of the static mixer. The membrane overlapped the walls of the defect by at least $2 \mathrm{~mm}$. The thickness was approximately $1 \mathrm{~mm}$ (Fig $2 \mathrm{~b}$ ). No fixation was needed as the gel adheres to the surrounding tissues (Fig.3)

\section{Wound closure}

The mucoperiosteal flap was sutured tightly and tension free over the membrane. The type of suture (e.g. needle shape, suture material, diameter) and technique was performed according to the clinical routine of the individual study center. Only submerged healing of the implant was allowed. The wound closure should be sufficient to completely cover the membrane. Membranes, which could not be covered had to be reported as an adverse event.

\section{Postoperative procedures}

It was recommended to take a radiograph. Antibiotic coverage or analgesics could be given as required and had to be recorded in the concomitant medication form in the CRF. Patients were instructed not to brush in the treated area and were instructed to rinse with chlorhexidine 
for plaque control.

\section{Re-entry}

After six months of submerged healing, access flap surgery was performed according the surgeon's standard procedure to assess the grade of bone regeneration. Following minor flap elevation, the vertical bone height was recorded (Fig. 4). Subsequently, cover screws were removed and healing abutments were applied according to soft tissue conditions (Fig. 5). Necessary soft tissue corrections or additional grafting was recorded.

\section{Statistical analysis and sample size calculation}

The statistical analysis was conducted using SPSS statistical software version 21 from IBM. Prior to the study, sample size calculation was performed for the primary outcome and a noninferiority test on the basis of a previously performed single centre clinical study (Jung et al. 2009) which showed a mean ( \pm standard deviation) difference in relative changes of defect height between $\mu \mathrm{T}$ (test) and $\mu \mathrm{S}$ (control) of $-1.5 \pm 8.5 \%$. Given a non-inferiority limit of $5 \%$ a sample size of 94 patients (47 per group) would have $88 \%$ power in a two group one-sided ttest with $\alpha=0.05$ or a $90 \%$ confidence interval. Taking into account a drop-out rate of more than $20 \%$ for the per-protocol analysis, a total sample size of 118 patients would be needed.

Descriptive statistics (mean, standard deviation, median, $25 \%$ and $75 \%$ quartiles, minimum and maximum) were recorded for each variable and group including the secondary outcomes (dehiscence comparison, keratinezed gingiva, implant failure). The non-inferiority of the relative changes of defect height were tested using a two-sided $90 \%$ confidence interval. Additionally, within-group comparisons for relative changes of defect height were performed using a paired t-test. The comparison of the independent two group means is based on the ttest, because the sample sizes are large. Adverse events were summarized with respect to BG and PEG groups. Results were found significant at $p<0.05$. 


\section{Results}

A total of $n=117$ patients (PEG $n=60, B G n=57)$ ( 80 female/ 37 male) with a mean age of 48.8 \pm 13.7 years were treated in 9 study centers. Patient recruitment resulted in a heterogeneous distribution within the study centers ( $n=5-21$ patients). A total of $n=114$ patients completed the observation period at 6 months (PEG $n=57, B G n=57$ ). Three patients of the PEG group were excluded prematurely due to wound infection and subsequent implant removal.

\section{Primary parameter}

The primary efficacy variable in this clinical study was the vertical defect height assessed at surgery (visit 2) compared to 6 months later (visit 7). The mean initial defect height was 4.0 $\mathrm{mm}$ in the PEG group and $4.6 \mathrm{~mm}$ in the BG group. After six months, the mean initial defect height decreased to $1.7 \mathrm{~mm}$ in the PEG group and to $1.5 \mathrm{~mm}$ in the $B G$ group (table 2). When categorized, the initial defect size ranged from 3 to $12 \mathrm{~mm}$ deep defects. The mean of absolute reduction in defect size amounted to $2.5 \mathrm{~mm}$ in the PEG group and $3.2 \mathrm{~mm}$ in the BG group (table 3). These changes were statistically significant $(p<0.001)$. The difference of defect size reduction was not statistically significant between the two treatment groups $(p=0.076)$. The absolute mean reduction in defect size ranged from $1.8 \mathrm{~mm}$ to $3.6 \mathrm{~mm}$ in eight study centers. In one center ( $\mathrm{n}=17$ patients) this effect was more pronounced in patients who were treated with PEG compared to patients treated with BG.

In addition to the absolute defect height changes in $\mathrm{mm}$, the relative changes in defect height displayed in percentage have been analysed in the present study. Hence, the mean of the relative change in defect height was $59.7 \%$ in the PEG group and $64.4 \%$ in the BG group (table 4). Both changes were statistically significant, $p<0.001)$. The difference in relative defect size reduction are not statistically significant between the two treatment groups $(p=0.474)$. The lower limit of $90 \%$ two-sided confidence interval is $-15.4 \%$, lower than the inferiority limit of $5 \%$. Hence, the non-inferiority could not be shown. The relative mean reduction in defect size ranged from $40.5 \%$ to $77.1 \%$ in eight study centers. Figure $6 a-d$ illustrate various re-entries of 
groups PEG an BG.

\section{Secondary parameters}

Crestal bone level values and changes at baseline, 6 months and 18 months are presented in tables 4 and 5. The mean mesial and distal values were equal at baseline. Therefore, measurements are presented as an average over both localisations (mesial and distal values were summarized for the comparison). In both groups, the increase in crestal bone levels was statistically significant $(p<0.001)$ at the follow-up time points. The lower limit of $90 \%$ confidence interval is -0.21 .

The physical appearance of the soft tissue was normal in all patients at visit 1 . The soft tissue was swollen in $29.7 \%$ in all patients in the PEG group and $26.8 \%$ in the BG group at suture removal (visit 3). The form of the soft tissue normalized during the further healing period. The colour of the soft tissue was pink in $75.7 \%$ of the patients in the PEG group and $80 \%$ in the BG group at the beginning of the study (visit 1). Some patients revealed soft tissue categorized as red. The percentage of red soft tissue was increased at suture removal (visit 3 ) in both treatment groups with values of $40.5 \%$ in the PEG and $46.3 \%$ in the BG group. The colour changed to pink during further healing.

The absolute numbers of soft tissue dehiscences are presented in table 7. Dehiscences were particularly observed at visit 3 (suture removal/7-10 days) and visit 4 (study day 12-14). Within this healing period, 4 patients treated with PEG and 8 patients treated with BG were associated with soft tissue dehiscences, while the incidence decreased during the course of the study. Eventhough the absolute frequencies were higher in the BG group these differences did not reach statistical significance $(p>0,05)$.

The height of the keratinized mucosa decreased constantly in both treatment groups during the course of the study (table 8 ). The initial keratinized mucosa height was $2.8 \mathrm{~mm}$ in the PEG group and $3.7 \mathrm{~mm}$ in the BG group. It remained rather constant until visit 7 (6 months) and decreased during follow-up until $1.8 \mathrm{~mm}$ in the PEG group and 2.4 in the BG group.

Implant survival was $100 \%$ in the BG group, whereas in the PEG group three implants were 
lost, one implant due to dehiscence and $6 \mathrm{~mm}$ probing depth and two implants were removed due to infection of the augmented area, resulting in an implant survival of $95 \%$.

\section{Adverse events}

An adverse event was defined as any medical occurrence in a patient which does not necessarily have a cause relationshiop with the treatment. A total of 30 adverse events out of the 117 treated patients $(20.5 \%)$ were noted of which three adverse events were related to the study device (delayed wound healing or infection). 24 of these 30 advese events occurred during the first 6 months the other at the follow-up visits. The type of adverse events varied largely: infection, inflammation, periimplantitis, delayed epithelialization, swelling, allergy, dehiscence defect, lack of osseointegration, pain, cancer, cerebral infarcation, paresthesia) The treatments had to be adjusted according to the type of adverse events.

From the 30 events, more patients were affected in the PEG group ( $30 \%$ of all PEG patients) compared to the BG group (10.5\% of all BG patients).

\section{Soft tissue corrections}

Soft tissue corrections were necessary at visit 7 (6 months post surgery) in 7 patients (6.2\%, 4 patients of the PEG group, 3 patients of the BG group). Either a connective tissue (6 cases) or a free gingival graft (1 case) was performed. 


\section{Discussion}

The present prospective, controlled, randomized, clinical multicenter trial showed that both investigated barrier membranes supported bone regeneration at dehiscence-type defects around titanium implants after 6 months of healing. At the follow-up visit 18 months after loading no statistically significant difference could be found in any clinical and radiographic parameter comparing the two membrane types.

Both groups, either the PEG membrane or the native collagen membrane showed a comparable vertical bone fill revealing a relative change in defect height of $59,7 \%$ and $64,4 \%$, respectively. These values are in accordance with previous studies on guided bone regeneration at dehiscence-type defects using different bone substitute materials and barrier membranes, reporting a vertical bone fill of $54-97 \%$ after $4-7.7$ months of healing (Jensen \& Terheyden, 2009). Recently, a randomized, controlled clinical trial using a similar study protocol demonstrated that a synthetic PEG membrane was successfully used as a biodegradable barrier membrane for GBR in humans (Jung, et al., 2009). This was documented by a similar amount of defect resolution compared with defects treated with a native collagen membrane (Jung, et al., 2009). At initial defect heights ranging from 4.5 to $6 \mathrm{~mm}$, the study reported of a vertical bone fill of $94.9 \%$ at sites treated with the PEG membrane and $94.4 \%$ in the group treated with a native collagen membrane (Jung, et al., 2009). These percentages of vertical bone fill are higher compared to the values of the present study. The methodological difference of these two studies is related to the graft material that has been used. In the former study (Jung, et al., 2009) a deproteinized bovine bone mineral (DBBM) has been used while the present study used an alloplastic biphasic calcium phosphate. Whether or not the faster resorbing alloplastic material is responsible for the lower rate of vertical bone fill remains unclear. An additional difference between these two clinical studies was the type of implant that has been used for each individual study. The present study used bone level type implants whereas the former study (Jung, et al., 2009) used soft tissue level type of implants. It might be speculated that it is easier to achieve a complete bone fill around 
a soft tissue level implant because a $100 \%$ bone fill is reached at the rough/smooth border of the implant which is $1.8 \mathrm{~mm}$ more apical to the implant shoulder. For a bone level implant a $100 \%$ bone fill is achieved when the bone is at the level of the implant shoulder.

In the present study, $51.8 \%$ of all subjects reached almost a complete absolute vertical defect fill $(0-1 \mathrm{~mm}$ residual defect height), which is similar to lower than previous investigations revealing an average of $67.7 \%$ (Jensen \& Terheyden, 2009). The initial defect height ranged from 3 to $12 \mathrm{~mm}$ in both groups, while mean defect height prior GBR treatment was $4.3 \mathrm{~mm}$. The absolute mean reduction in defect size amounted to $2.5 \mathrm{~mm}$ in the PEG group and 3.2 $m m$ in the BG group. Although the mean defect reduction was statistically significant $(p<0.001)$, the difference of defect size reduction did not show a statistically significant difference between the two treatment groups $(p=0.076)$. In the PEG group, mean residual defect height was $1.7 \mathrm{~mm}$ compared to $1.6 \mathrm{~mm}$ in the BG group. Results from comparable studies and the present investigation examining GBR with various bone substitute materials and different barrier membranes show that complete defect regeneration is infrequently achieved (Annen, Ramel, Hammerle \& Jung, 2011; Becker, et al., 2009; Hammerle \& Lang, 2001; Jung, et al., 2009; Zitzmann, Naef \& Scharer, 1997). A long-term assessment over 4 and 8 years following GBR at dehiscence-type defects pointed to a correlation between residual defect height $(R D H)$ and the incidence of periimplant lesions (Schwarz, Sahm \& Becker, 2012b; Schwarz, Schmucker \& Becker, 2017). In the presence of $\mathrm{RDH}$ values of 1 or $\geq 1 \mathrm{~mm}$, mean mucosal recession values were twice as much as at sites with complete defect fill (Schwarz et al. 2012, (Schwarz, et al., 2017). Moreover, it has been shown that $\mathrm{RDH}$ values $>1 \mathrm{~mm}$ were at higher risk of developing periimplant diseases, especially at implant sites showing high baseline RDH values of 4 to $6 \mathrm{~mm}$ (Schwarz, et al., 2012b). In the present study no higher incidence of periimplant lesions have been recorded after an observation period of 18 months. Further long-term data are necessary to monitor this important issue.

As secondary parameters, crestal bone levels and soft tissue conditions, including keratinized mucosa as well as the occurence of dehiscences and physical appearance of the study site were evaluated. Radiographic evaluation of crestal bone levels indicated that implants were 
well osseointegrated. Mean MBL amounted to $0.53 \pm 0.43 \mathrm{~mm}$ for the PEG group and to 0.49 $\pm 0.65 \mathrm{~mm}$ for the BG group after 6 months of loading. After 18 months, mean MBL were 0.67 \pm 0.46 in the PEG group and $0.75 \pm 1.00$ in the BG group. The difference between baseline and the follow-up time points was statistically significant $(p<0.001)$ showing a slight increase over 18 months. These values are in accordance with previous clinical studies evaluating marginal bone level changes at implants inserted in pristine bone or with simultaneous GBR (Benic, Jung, Siegenthaler \& Hammerle, 2009; Mayfield, Skoglund, Nobreus \& Attstrom, 1998; Zitzmann, Scharer \& Marinello, 2001). In a controlled study, a mean radiographic bone loss of $0.7 \mathrm{~mm}$ for implants with GBR was observed $23-27$ months following prosthetic loading (Mayfield, et al., 1998). Another clinical study comparing the crestal aspect at implants in regenerated or native bone demonstrated $\mathrm{MBL}$ values of 1.3-1.6 mm after a 5-year follow-up examination (Benic, et al., 2009). Thus, marginal bone levels in the present study were within the range of values reported previously.

At suture removal, dehiscences appeared in $18.3 \%$ in the PEG group and $17.5 \%$ in the BG group. At 12-14 days after surgery, both treatment groups revealed dehiscence percentages of $17.9 \%$. The incidence decreased during the course of the study. These observations are in accordance with previous clinical data on the same GBR model and membrane combinations (Jung, et al., 2009). At the time of suture removal, they reported soft tissue dehiscences at PEG sites in $31 \%$ and at BG sites in $22 \%$ of the cases. Another clinical study comparing two different collagen membranes with an ePTFE membrane described a similar pattern of wound healing reporting $32.1 \%$ wound dehiscence at the time of suture removal (Moses, Pitaru, Artzi \& Nemcovsky, 2005). In both studies, authors reported of impaired soft tissue healing at these sites resulting in exposure of the cover screw (Jung, et al., 2009) or significant decrease in the amount of defect reduction (Moses, et al., 2005). In the present study, 15.8\% of the PEG sites and $10.5 \%$ of the BG sites did not heal completely at re-entry after 6 months. In cases with premature membrane exposure, sites were commonly associated with impaired bone regeneration in both PEG and BG groups showing reduced values for absolute defect reduction ( $26.5 \%$ and $47.3 \%$, respectively). 
Despite the promising data of the present study supporting bone regeneration using the PEG membrane, some limitations need to be addressed. The present clinical data investigated on relatively small dehiscence-type defects which makes it impossible to draw any conclusion for larger bone defects. Although preclinical data described promising outcomes using PEG for saddle-type defects, there is no controlled clinical data available for extensive bone defects. Moreover, the way of applying the PEG membrane might influence clinical outcomes. As every case is applied individually there is always variablity in PEG membrane thickness, especially in comparison to prefabricated native collagen membranes. Excessive membrane volume might influence the biodegradation pattern or lead to mechanical stress of the overlying soft tissue resulting in wound healing complications.

\section{Conclusion}

Both membranes supported bone regeneration at dehiscence-type defects and obtained vertical bone fill with a relative change in defect height of $59.7 \%$ (PEG) and $64.4 \%$ (BG), however the non-inferiority of PEG could not be shown. The PEG membrane revealed more soft tissue complications compared to the BG membrane without statistically significant differences. 


\section{References}

Annen, B. M., Ramel, C. F., Hammerle, C. H. \& Jung, R. E. (2011). Use of a new cross-linked collagen membrane for the treatment of peri-implant dehiscence defects: a randomised controlled double-blinded clinical trial. European Journal of Oral Implantology, 4, 87-100.

Becker, J., Al-Nawas, B., Klein, M. O., Schliephake, H., Terheyden, H. \& Schwarz, F. (2009). Use of a new cross-linked collagen membrane for the treatment of dehiscence-type defects at titanium implants: a prospective, randomized-controlled double-blinded clinical multicenter study. Clinical Oral Implants Research, 20, 742-749. doi:10.1111/j.1600-0501.2008.01689.x

Benic, G. I. \& Hammerle, C. H. (2014). Horizontal bone augmentation by means of guided bone regeneration. Periodontology 2000, 66, 13-40. doi:10.1111/prd.12039

Benic, G. I., Jung, R. E., Siegenthaler, D. W. \& Hammerle, C. H. (2009). Clinical and radiographic comparison of implants in regenerated or native bone: 5-year results. Clinical Oral Implants Research, 20, 507-513. doi:10.1111/j.1600-0501.2008.01583.x

Gottlow, J., Laurell, L., Teiwik, A. \& Genon, P. (1994). Guided tissue regeneration using a bioresorbable matrix barrier. Practical Periodontics and Aesthetic Dentistry, 6, 71-78; quiz 80.

Hammerle, C. H. \& Lang, N. P. (2001). Single stage surgery combining transmucosal implant placement with guided bone regeneration and bioresorbable materials. Clinical Oral Implants Research, 12, 9-18. doi:10.1034/j.1600-0501.2001.012001009.x

Herten, M., Jung, R. E., Ferrari, D., Rothamel, D., Golubovic, V., Molenberg, A., Hammerle, C. H., Becker, J. \& Schwarz, F. (2009). Biodegradation of different synthetic hydrogels made of polyethylene glycol hydrogel/RGD-peptide modifications: an immunohistochemical study in rats. Clinical Oral Implants Research, 20, 116-125. doi:10.1111/j.1600-0501.2008.01622.x

Jensen, S. S. \& Terheyden, H. (2009). Bone augmentation procedures in localized defects in the alveolar ridge: clinical results with different bone grafts and bone-substitute materials. International Journal of Oral and Maxillofacial Implants, 24 Suppl, 218-236.

Jung, R. E., Benic, G. I., Scherrer, D. \& Hammerle, C. H. (2015). Cone beam computed tomography evaluation of regenerated buccal bone 5 years after simultaneous implant placement and guided bone regeneration procedures--a randomized, controlled clinical trial. Clinical Oral Implants Research, 26, 28-34. doi:10.1111/clr.12296

Jung, R. E., Fenner, N., Hammerle, C. H. \& Zitzmann, N. U. (2013). Long-term outcome of implants placed with guided bone regeneration (GBR) using resorbable and non-resorbable membranes after 12-14 years. Clinical Oral Implants Research, 24, 1065-1073. doi:10.1111/j.1600-0501.2012.02522.x 
Jung, R. E., Halg, G. A., Thoma, D. S. \& Hammerle, C. H. (2009). A randomized, controlled clinical trial to evaluate a new membrane for guided bone regeneration around dental implants. Clinical Oral Implants Research, 20, 162-168. doi:10.1111/j.1600-0501.2008.01634.x

Jung, R. E., Zwahlen, R., Weber, F. E., Molenberg, A., van Lenthe, G. H. \& Hammerle, C. H. (2006). Evaluation of an in situ formed synthetic hydrogel as a biodegradable membrane for guided bone regeneration. Clinical Oral Implants Research, 17, 426-433. doi:10.1111/j.16000501.2005.01228.x

Mayfield, L., Skoglund, A., Nobreus, N. \& Attstrom, R. (1998). Clinical and radiographic evaluation, following delivery of fixed reconstructions, at GBR treated titanium fixtures. Clinical Oral Implants Research, 9, 292-302. doi:10.1034/j.1600-0501.1998.090502.x

Mihatovic, I., Becker, J., Golubovic, V., Hegewald, A. \& Schwarz, F. (2012). Influence of two barrier membranes on staged guided bone regeneration and osseointegration of titanium implants in dogs. Part 2: augmentation using bone graft substitutes. Clinical Oral Implants Research, 23, 308-315. doi:10.1111/j.1600-0501.2011.02238.x

Moses, O., Pitaru, S., Artzi, Z. \& Nemcovsky, C. E. (2005). Healing of dehiscence-type defects in implants placed together with different barrier membranes: a comparative clinical study. Clinical Oral Implants Research, 16, 210-219. doi:10.1111/j.1600-0501.2004.01100.x

Nyman, S. R. \& Lang, N. P. (1994). Guided tissue regeneration and dental implants. Periodontology 2000, 4, 109-118. doi:10.1111/j.1600-0757.1994.tb00011.x

Ramel, C. F., Wismeijer, D. A., Hammerle, C. H. \& Jung, R. E. (2012). A randomized, controlled clinical evaluation of a synthetic gel membrane for guided bone regeneration around dental implants: clinical and radiologic 1- and 3-year results. International Journal of Oral and Maxillofacial Implants, 27, 435-441.

Rothamel, D., Schwarz, F., Sager, M., Herten, M., Sculean, A. \& Becker, J. (2005). Biodegradation of differently cross-linked collagen membranes: an experimental study in the rat. Clinical Oral Implants Research, 16, 369-378. doi:10.1111/j.1600-0501.2005.01108.x

Schwarz, F., Mihatovic, I., Golubovic, V., Hegewald, A. \& Becker, J. (2012a). Influence of two barrier membranes on staged guided bone regeneration and osseointegration of titanium implants in dogs: part 1. Augmentation using bone graft substitutes and autogenous bone. Clinical Oral Implants Research, 23, 83-89. doi:10.1111/j.1600-0501.2011.02187.x

Schwarz, F., Rothamel, D., Herten, M., Sager, M. \& Becker, J. (2006). Angiogenesis pattern of native and cross-linked collagen membranes: an immunohistochemical study in the rat. Clinical Oral Implants Research, 17, 403-409. doi:10.1111/j.1600-0501.2005.01225.x

Schwarz, F., Sahm, N. \& Becker, J. (2012b). Impact of the outcome of guided bone regeneration in dehiscence-type defects on the long-term stability of peri-implant health: clinical observations at 4 years. Clinical Oral Implants Research, 23, 191-196. doi:10.1111/j.1600-0501.2011.02214.x 
Schwarz, F., Schmucker, A. \& Becker, J. (2017). Long-term outcomes of simultaneous guided bone regeneration using native and cross-linked collagen membranes after 8 years. Clinical Oral Implants Research, 28, 779-784. doi:10.1111/clr.12881

Zitzmann, N. U., Naef, R. \& Scharer, P. (1997). Resorbable versus nonresorbable membranes in combination with Bio-Oss for guided bone regeneration. International Journal of Oral and Maxillofacial Implants, 12, 844-852.

Zitzmann, N. U., Scharer, P. \& Marinello, C. P. (2001). Long-term results of implants treated with guided bone regeneration: a 5-year prospective study. International Journal of Oral and Maxillofacial Implants, 16, 355-366. 


\section{Figure legends}

Fig. 1 a) Schematic graphic showing the measurement of the periimplant dehiscence defect from the implant shoulder to the deepest bone-to-implant contact.

b) Clinical photograph illustrating the measurement of the dehiscence defect at the implant

Fig. 2 a) Photograph series showing the dehiscence defect at the implant, the application of the bone substitute material and the placement of the collagen membrane (BG group)

b) Photograph series showing the dehiscence defect at the implant, the application of the bone substitute material and the placement of the PEG membrane (PEG group)

Fig. 3 Final contouring of the PEG memrane according to the study protocol. The membrane overlapped the walls of the defect by at least $2 \mathrm{~mm}$. The thickness was approximately $1 \mathrm{~mm}$. No fixation was needed as the gel adheres to the surrounding tissues

Fig. 4 Measurement of the the residual defect at re-entry

Fig. 5 Healing abutment connection and readaptation of the soft tissue with sutures.

Fig. 6 Photograph series showing the dehiscence defect after implant placement and bone regeneration at re-entry in different groups (a: BG group, b: PEG group, c: PEG group, d: PEG group) 


\section{Tables}

Table 1: Demographic data (mean \pm SD) at baseline $(n=117$ patients)

\begin{tabular}{lccc} 
& Age (years) & Female & Male \\
\hline PEG & $49.6 \pm 13.6$ & 46 & 14 \\
BG & $47.8 \pm 13.9$ & 34 & 23
\end{tabular}

Table 2: Defect heights (mean $\pm \mathrm{SD}$ ) at surgery and at re-entry after 6 months, with mean and its SD of the differences of PEG - BG

\begin{tabular}{lcc} 
& At surgery & 6 months \\
\hline PEG & $4.0 \pm 0.9$ & $1.7 \pm 1.4$ \\
BG & $4.6 \pm 1.8$ & $1.5 \pm 1.1$ \\
Diff & $-0.6 \pm 0.3$ & $0.2 \pm 0.3$
\end{tabular}

Table 3: Absolute change in defect height after 6 months (mean \pm SD), with mean and its SD of the differences of PEG - BG

\begin{tabular}{lc} 
& 6 months \\
\hline PEG & $2.5 \pm 1.5$ \\
BG & $3.2 \pm 2.1$ \\
Diff & $-0.7 \pm 0.4$
\end{tabular}

* PEG after 6 months: $t=9.05, d f=39, p<0.001, B G$ after 6 months: $t=9.50, d f=44, p<0.001$ Comparison of the differences to visit 2 between the groups after 6 months: $t=-1.80, d f=83$, $\mathrm{p}=0.076 /$ mean diff. $=-0.7278,95 \% \mathrm{Cl}=-1.534$ to 0.078

Table 4: Relative change (mean \pm SD) in defect height $(\%)$, with mean and its SD of the differences of PEG - BG

\begin{tabular}{lc} 
& 6 months \\
\hline PEG & $59,7^{*} \pm 32,5$ \\
BG & $64,4^{*} \pm 27,2$ \\
\hline Diff & $-4.7 \pm 6.5$
\end{tabular}


${ }^{*} p<0.001$. The difference in relative defect size reduction is not statistically significant between the two treatment groups $(p=0.474)$. The lower limit of the $90 \%$ two-sided confidence interval for the difference of the means at 6 months is $-15.4 \%$. Non-inferiorty cannot be shown since the lower limit is lower than the non-inferiority limit of $-5 \%$.

Table 5: Crestal bone level measurements (mesial+distal) in mm (mean $\pm \mathrm{SD})$, with mean and its SD of the differences of PEG - BG

\begin{tabular}{lccc} 
& Baseline & 6 months & 18 months \\
\hline PEG & $0.25 \pm 0.16$ & $0.54 \pm 0.43$ & $0.67 \pm 0.46$ \\
BG & $0.26 \pm 0.29$ & $0.50 \pm 0.65$ & $0.75 \pm 1.00$ \\
Diff & $-0.01 \pm 0.05$ & $-0.04 \pm 0.13$ & $-0.08 \pm 0.17$
\end{tabular}

Table 6: Crestal bone level change compared to baseline (mesial+distal) in $\mathrm{mm}$, with mean and its SD of the differences of PEG - BG

\begin{tabular}{lcc} 
& 6 months & 18 months \\
\hline PEG & $0.32 \pm 0.45$ & $0.45 \pm 0.43$ \\
BG & $0.29 \pm 0.66$ & $0.41 \pm 0.81$ \\
Diff & $0.03 \pm 0.14$ & $0.04 \pm 0.15$
\end{tabular}

* In both groups, the increase in crestal bone levels was statistically significant $(p<0.001)$ for both time points. The two groups did not show significant differences of the means $(p>0.79)$. The lower limit of the $90 \%$ two-sided confidence interval of the difference of the means is -0.21 at 6 months and -0.21 at 18 months. 
Table 7: Number of patients with dehiscence at different healing phases

12-14 6-7 18 7-10 days days 4 weeks 3 months 6 months months months

\begin{tabular}{llllllll}
\hline PEG & 4 & 4 & 3 & 3 & 2 & 1 & 1 \\
BG & 8 & 8 & 7 & 6 & 4 & 0 & 0
\end{tabular}

* Differences between groups were not statistically significant $(p>0.05)$

PPD Data:

Table 8: Height of keratinized mucosa at different healing phases (mean $\pm S D$ ), with mean and its $S D$ of the differences of PEG - BG

$\begin{array}{llllllll}\text { Baseline } & \text { Visit } 3 & \text { Visit } 4 & \text { Visit } 5 & \text { Visit } 6 & \text { Visit } 7 & \text { Visit } 8 & \text { Visit } 9\end{array}$

PEG $\quad 2.8 \pm 1.3 \quad 2.6 \pm 1.3 \quad 2.9 \pm 1.4 \quad 2.6 \pm 1.3 \quad 2.6 \pm 1.2 \quad 2.6 \pm 1.3 \quad 2.2 \pm 1.0 \quad 2.1 \pm 0.9$

BG $\quad 3.7 \pm 1.4 \quad 3.3 \pm 1.2 \quad 3.2 \pm 1.2 \quad 3.0 \pm 1.3 \quad 3.0 \pm 1.2 \quad 3.1 \pm 1.4 \quad 2.6 \pm 1.2 \quad 2.4 \pm 1.2$

Diff. $\quad-0.9 \pm 0.3 \quad-0.7 \pm 0.1 \quad-0.3 \pm 0.3 \quad-0.4 \pm 0.3 \quad-0.4 \pm 0.3 \quad-0.5 \pm 0.3 \quad-0.4 \pm 0.3 \quad-0.3 \pm 0.2$ 


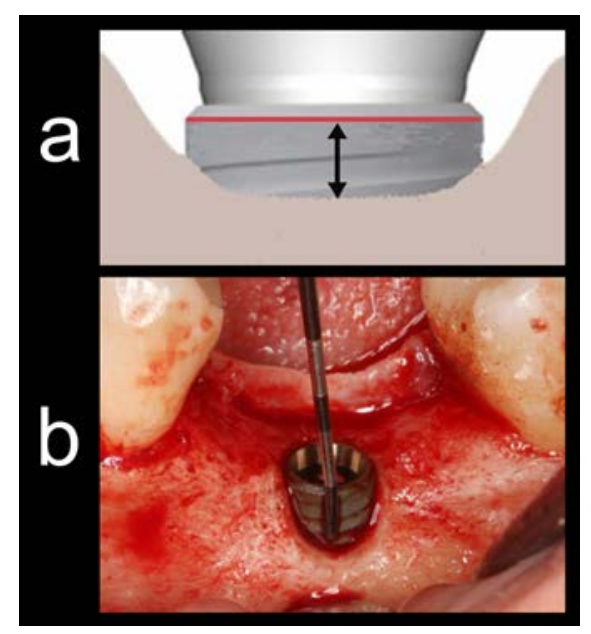

Figure 1

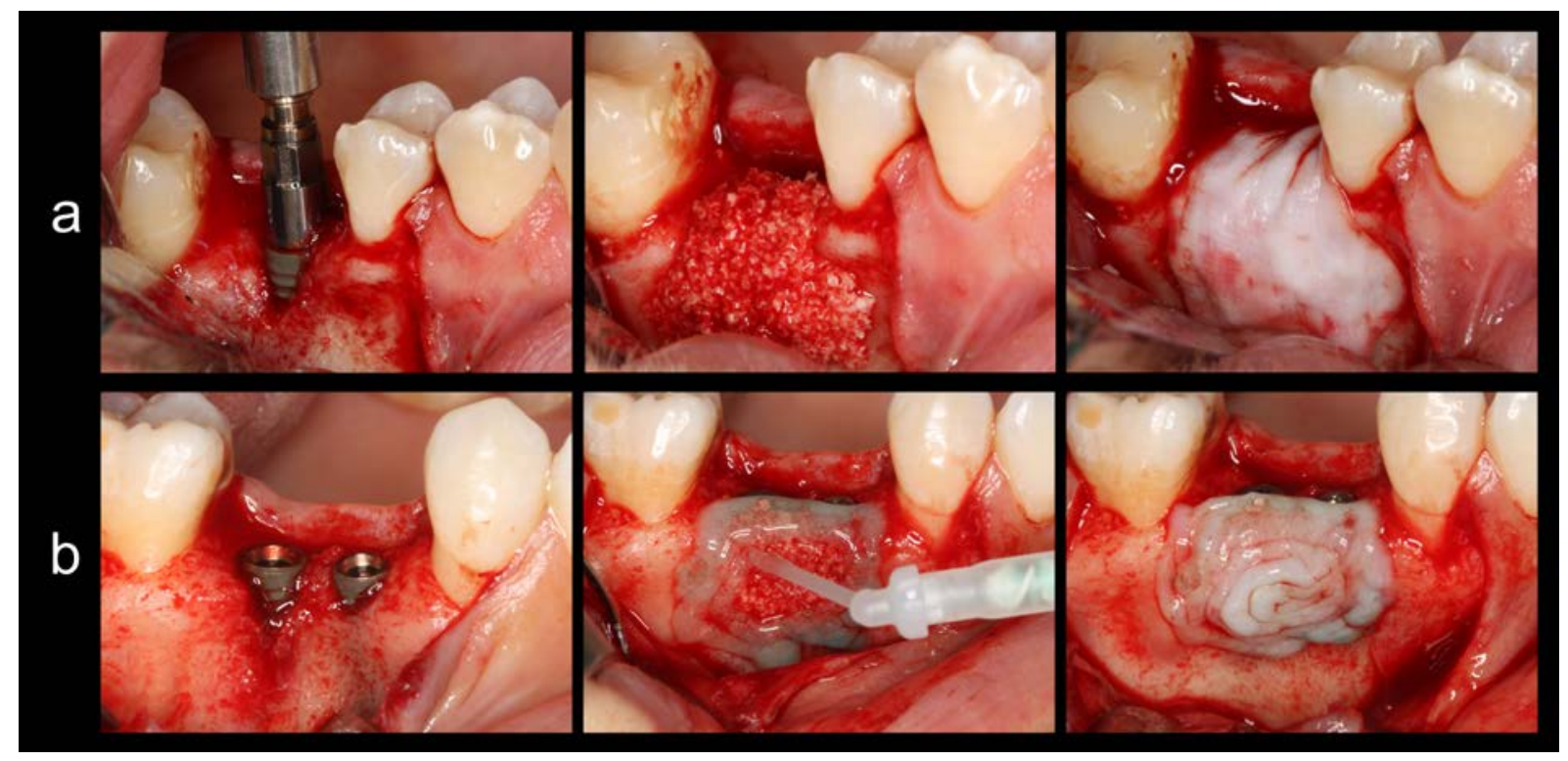

Figure 2

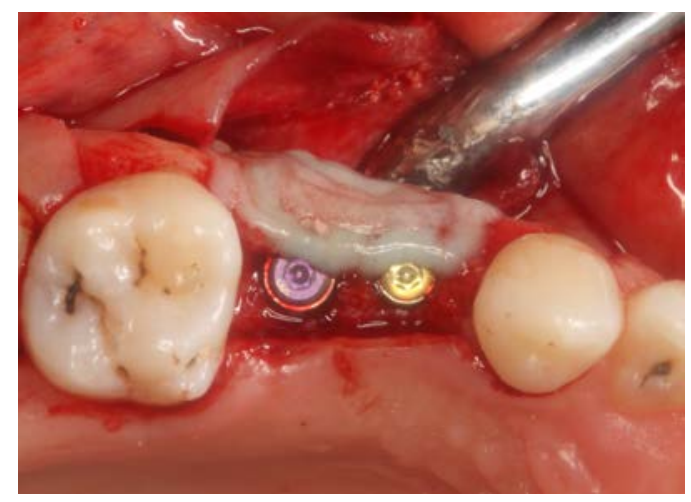

Figure 3 


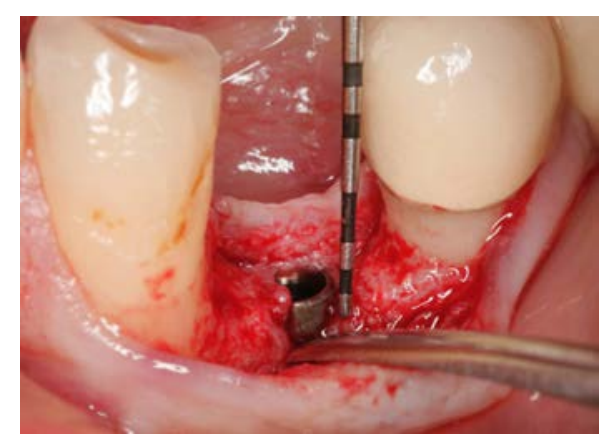

Figure 4

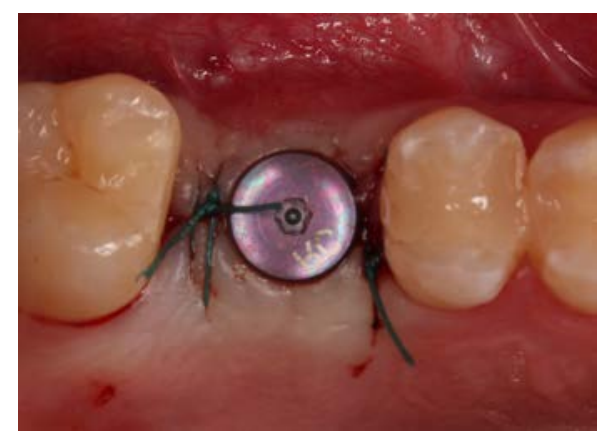

Figure 5

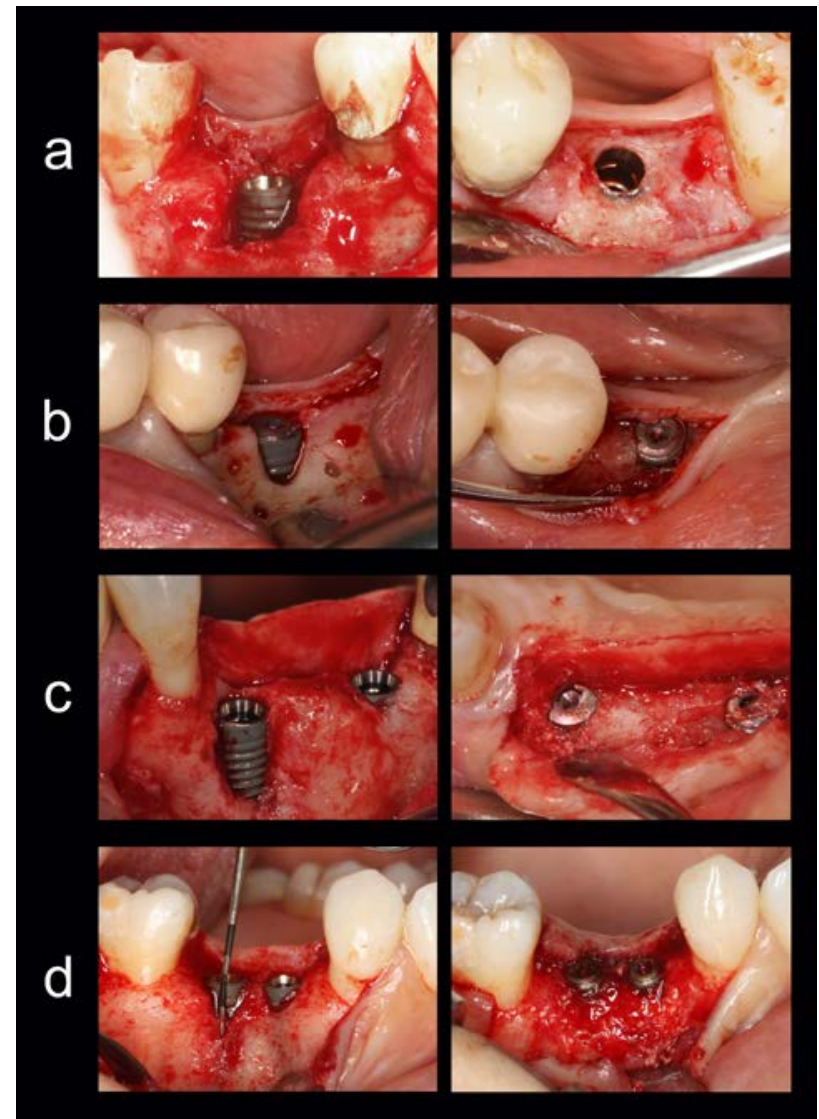

Figure 6 